With specific ideas drawing from the diverse perspectives of its authors, this chapter describes opportunities that extend beyond advising to mentoring for those undergraduates who aspire to careers in the health professions. Although issues in the health sciences mirror those in education in general, the mentoring network described here utilizes individuals who can provide unique insights for students interested in the health professions.

\title{
Mentoring for the Health Professions
}

\author{
Timothy R. B. Johnson, Philip D. Settimi, \\ and Juliet L. Rogers
}

In 1977, John Bruhn wrote "[p]remedicine and medicine are worlds apart. ... There is an acute need to revise premedical advising to better meet the needs of students. Medical schools should share the responsibility and initiatives in creating a system whereby career choices are made by positive actions" (Bruhn, 1977, p. 678). Despite Bruhn's plea, we believe there has been no systematic improvement or change in the premedical advising process, much less for those with nonmedical prehealth interests, in the last twenty-five years. Opportunities abound for improvements in appropriate-career selection, preprofessional preparation, and facilitation and improvement of the application process for potential premedical and other prehealth students. We hope the current interest in mentorship can focus on neglected issues and lead to a more satisfying process for individuals as well as more appropriate societal distribution of new, intelligent service providers.

College advisers, in addition to traditional academic advising, have the opportunity to improve and enrich prehealth students' growth by also becoming mentors, encouraging and enabling students to identify other mentors, and working to develop an effective and informed mentor network. Mentoring is a teaching opportunity to guide students as they explore their nonacademic interests and their values. Mentors with insights about the joys and challenges in health professions are well positioned to help students evaluate their choices and understand the far-reaching consequences of those choices. The mentor does not give all the answers but instead helps students begin to pose all the questions. 


\section{Career Selection}

There is an increasing variety of health professions and health-related careers available to students. Many undergraduate students begin as premedical students not realizing that other satisfying careers are available or that these other careers might be either preferable from a personal point of view or more realistically attainable. Ensuring that students have adequate exposure to opportunities in nursing, social work, public health, and related health professions (physical therapy, respiratory therapy, occupational therapy, music and dance therapy) is increasingly appropriate given the fierce premedical competition. Linked with this is the importance of reality testing for those students for whom medical school is not a viable option. Early encouragement of these students includes presenting them with a broad potential list of opportunities. Challenges will remain in reaching those students who do not hear the advice given or fail to use existing advising services (Trevino, Fuentes, and Bruhn, 1977).

\section{Roles for the Mentor}

Mentors should be readily accessible to observe the decision-making process and offer suggestions to guide it. The best mentors will never attempt to make decisions for the student. Ideally a mentor-student relationship can be founded on similar professional, academic, or community interests. It can be of assistance, though not necessary, for mentors to be well-known in their community and to be current with the job-search or grad-school-research process. It may only be necessary for students to meet with their mentors two to four times annually. While that may match the number of visits to the Undergraduate Advising Office, the quality of the meeting may be higher given a more relaxed, focused atmosphere. In any case, the simple fact that the mentor does not have to spend time with many other students or be concerned with some academic issues should allow for a more personal relationship to develop.

After identifying a viable mentor, the student should be aware that the mentor's expertise is not described by which science classes are most difficult or how many to schedule in a semester. That is a job for the general prehealth adviser. Instead, a mentor will be able to listen to a student's thoughts, observe his or her activities outside of school, and offer suggestions about how to identify additional opportunities and long-term goals. For example, while recommending a standard hospital internship, a mentor might observe a student's interest in technology and identify biotechnology or bioengineering opportunities. A prehealth student might be advised to intern with a health economist or study health law.

It has been suggested that dual processes should be used, those of academic advising and separate career and professional counseling by either a preprofessional committee or an office of career planning and placement 
(Bruhn, 1977; Trevino, Fuentes, and Bruhn, 1977; Elam, Lenhoff, and Johnson, 1997). We believe local history and culture will determine institutional practice but that the issues and concerns we discuss should be systemically recognized and addressed.

An important role of the mentor is helping students develop an appropriate record not only academically but of preprofessional activities and community service. This will inevitably play a role in defining the students' career goals. Another important piece early on is assuming that the students are quite clear that they understand what medicine is, what public health is, and what the possible internships are. A variety of experiences are critical to allow students to explore opportunities and to pick the appropriate career paths. Once the appropriate and realistic career choice with viable and interesting alternative options is made, then the next step is easier: achievement of all the curricular, extracurricular, and community service activities that will maximize the student's candidacy.

\section{Curriculum or Classes and Grades}

The mentor should be generally aware of the curricular requirements and performance standards for acceptance to medical school and where more specific, reliable information can be obtained. The fact that most medical admission committees emphasize GPA and MCAT scores over noncognitive or community service achievements is clear (Trevino, Fuentes, and Bruhn, 1977; Hesser, Cregler, and Lewis, 1998). It is also clear that expectations from other health-professional schools may be less explicit and these should be sought from the school themselves.

Medical school admission officers and clinical faculty are also demonstrably interested in students with exposure to humanities and social sciences (Elam, Lenhoff, and Johnson, 1997; Imperato, 1997; Stimmel, Smith, and Kase, 1995). The fact that except for in biochemistry there is no benefit from electing advanced science courses that will reappear in the medical curriculum (Caplan, Kreiter, and Albanese, 1996) should lead to a broad curricular experience. Mentors could participate in and encourage this shift in concentration emphasis.

Opportunities for premedical, career-focused academic classes (Gerbens, Stid, and Foulds, 1998), seminars (Abdelman, Bryon, and Davidson, 1995), and hospital or premedical student internships (Alexander, Nevins, Lyon, Thayer, and Yere, 1983; Alexander, Lyon, Nevins, Yere, and Thayer, 1992) are all described and have been effective in helping students identify appropriate careers, and yet these competitive programs may be difficult to locate unless mentors remain continually vigilant for such opportunities. Specific opportunities for minority students after successful completion of summer academic enrichment programs have shown the programs' benefits (Hesser, Cregler, and Lewis, 1998; Bruhn, Fuentes, Trevino, and Williams, 1976). 


\section{Application}

After a career has been selected, and the appropriate internships, academic prerequisites, and personal growth have been identified, the application process needs attention. "Packaging" the applicant and ensuring appropriate "etiquette" during the application or admission process can maximize the candidate's success. The preprofessional committee or the professional counseling office often collects materials and makes summary recommendations that carry great weight. Mentors and institutions need to understand that academic applications are different from job applications; recommendations, dossiers, and evaluations should follow academic expectations. Students need advice about letters of recommendation. Too often these are requested of faculty with no knowledge of the values and perspective of medical or graduate program admission committees, even if they do have adequate knowledge of the students. Such letters often open with the fact that the student took their class and got a fine grade, and then go on to describe their class syllabus, their teaching goals, or their grading methods. Mentors should help students find and select recommendors and assist with the application package.

Unsuccessful applicants to medical school can and should have mentoring opportunities, optimally initiated before the rejection occurs, so they can quickly pursue attractive available options (Becker, Katatsky, and Seidel, 1973). Ideally, these students should have received adequate early counseling so that those likely to be admitted to medical school can reassess and reapply. Those less likely to achieve acceptance should have an alternative in place, which they actively pursue while applying to professional school. This prevents delay following rejection and permits early and satisfied selection of the predetermined alternative.

\section{Beyond the Medical School Option}

With appropriate academic and career advising, quality mentoring, and enlightening experiential learning opportunities, a considerable number of premedical undergraduates discover that medical school may not be the best fit for their interests or lifestyle goals. This is not where the advising relationship ends. Rather, it is where the greatest opportunity exists for the relationship to flourish. A plethora of career, graduate school, and employment opportunities exist for those students interested in health-science fields such as public health, health promotion, environmental health, gerontology, health management, movement science, midwifery, nursing, dental hygiene, and occupational and physical therapy.

For some students, these careers are the focus from the beginning and individualized, prehealth science advising should be offered to them in the first year. Other students have no exposure to and little knowledge of these areas of study and are introduced to them only after deciding that medical 
school is not for them or is out of reach for the time being. In either case, high-quality advice regarding the best course of study for prehealth science students, internship and career opportunities, professional societies, and accredited graduate school programs is often unavailable to these students. One reason for this is because the plans of study must be developed for each student based on the health-science area of interest. Graduate programs in occupational health have preparation and admission requirements that differ greatly from those of graduate programs in health-services management. Nonetheless, at the undergraduate level, students with very different interests and goals are often limited to the same academic advising optionsthose of the prehealth science advisers.

Some universities are trying to address this and related issues by offering one-hour workshops that are designed to introduce first- and secondyear students to a range of careers that may be of interest to those who are considering medical and nursing fields. The most helpful of these workshops will often feature panels of established professionals representing different interest areas and panelists who can articulate to students the many different paths (as opposed to set curricula) that students may choose at the undergraduate level if admission to graduate school is the end goal. Peer advising (another form of mentoring) that matches prehealth undergraduates with graduate students who are pursuing studies in the area of interest are often very helpful, especially at smaller schools where advisers with personal experience with health-science graduate programs may be in short supply.

\section{Innovative Opportunities for Mentorship}

It may not be until the third or fourth years of undergraduate study that some students discover their areas of interest. For these students, it is often difficult to complete their degrees in the regular time frame if a change in area of concentration or major course requirements is introduced as a result of their change in direction. Introducing students to the myriad of choices in the health-science professions at early stages in their undergraduate careers is one way to reduce the likelihood that this will happen for students interested in prehealth. The previous section identified career-planning workshops as one way to inform students of their options. Another way is to take the workshops to them, meeting them wherever they may be at that time. This can be achieved easily through curricular and experiential integration of "less traditional" health-science topics into the traditional health-science introductory courses. Undergraduate teachers should be encouraged to provide such mentorship by faculty administration and working with offices that also provide resources for successful implementation.

Take, for example, introductory biology. At larger universities, the introductory biology course for prehealth and science students may enroll 
five hundred students or more each term. Little time would be taken away, and much value would be added to the students' experience, if parts of one or several lectures included examples of how the theories or experiments related to the day's subject matter could be applied or expanded to fields of study with which students are less familiar. Instead of a discussion on how the physician or biologist would use the information, perhaps the students could be offered perspectives on the value of the information for the environmental health researcher or the dietician. To take this example further, consider the number of students who may be interested in hearing how the findings resulting from the biologist's experiment might help strengthen the argument of the women's health advocate or the health-policy analyst. The opportunities for this type of integration may not exist in each class session, but would certainly fit in a few over the course of the term.

Beyond health-science preparatory courses, many opportunities exist in social science and humanities curricula to introduce students to different fields of health-science study. Many women's-studies programs now feature courses in women's and reproductive health, health advocacy and activism, and the biology of women's cancers. English courses have been developed that emphasize the skills required for science and technical writing; others emphasize the power of the narrative for those with chronic health conditions. Sociology, American culture, and other social science and humanities departments increasingly are offering opportunities for students to gain exposure to lesser-known careers through experiential learning courses and community service projects. Experiential learning offers students on-the-job training, an environment in which to test their knowledge and apply their skills, and the opportunity to make valuable connections with potential future employers and reference writers.

Traditionally, established programs in experiential learning for prehealth science students were limited to "shadowing" or lab assistantships. Though both present a valuable opportunity for the aspiring doctor or medical researcher, they offer little to students who are interested in healthcareer options other than medical or research fields. Resources should now be directed to the development of complementary programs to better serve the interests of pre-public health students, while expanding the scope of the learning opportunities for premedical students. These programs should include experiential learning opportunities in government and private health advocacy and health-policy research agencies, schools, correctional facilities, community projects and social service programs, and other charities or nonprofits. Pre-public and allied-health students will gain valuable exposure to many career paths, premedical students will learn the importance of community health efforts in achieving wellness and preventing disease, and the community will benefit from the energy, enthusiasm, and project support of all students involved. 


\section{Redesigning the Advisory or Mentor Network}

In order to fulfill the complete support role for today's prehealth students, colleges and universities should consider emphasizing a coordinated and complementary advising system based on the use of traditional academic advisers and novel community- or university-affiliated mentors.

Under the traditional system of undergraduate advising for premedical students, now more broadly defined as prehealth students, a subset of the much larger advisory network supports an oftentimes unwieldy volume of students. With such a large percentage of undergraduates seeking prehealth advising, at many universities waiting times for advisers can be lengthy. In addition, appointments can be brief, impersonal, and consequently of suboptimal quality and use to the student. This outcome is not necessarily a direct consequence of any deficiencies on the part of the advisory network. Instead this scenario may simply represent a "necessary evil," whereby the sheer quantity of students and diversity of their backgrounds and educational experiences precludes their ability to receive high quality support for the full spectrum of challenges that faces prehealth students (for example, coursework, standardized tests, financial aid, extracurricular activities, or personal recommendations).

The current state of undergraduate prehealth advising appears to be simply a means to an end. The advising process is standardized for the purposes of maximizing efficiency and thus nearly identical packets of information and advice are delivered to tens of thousands of students annually. High-quality advising consists of personalized attention with up-to-date recommendations that truly "fit" the student. Identifying this "fit"-a representative picture of the student's interests as defined by the relative emphasis he or she places on academic, extracurricular, and vocational activities-is nearly impossible under the current status quo. Further, advisers' inability to diagnose this fit leads many to prescribe a protocol that is followed too closely by students who believe this advice holds the key to a graduateschool acceptance: volunteer at a local hospital, get research experience, shadow a doctor or nurse or veterinarian, and others on the all-too-familiar list. The current advisory system can place little emphasis on development of the individual. Thus a very unique student who has described his or her undergraduate experience in the "personal statement" on the medical school application begins to sound like the other hundreds of students who used identical advisory services.

That said, could colleges and universities realistically offer focused advice that is aligned with individual goals and unique interests of the student? The answer: probably not under the traditional advising system. There are a number of reasons that the current system is not able to provide information beyond that which can be easily found in any "Preparing for Grad School" book. The primary reason is logistics. It is simply unreasonable for three or four advisers to maintain personal relationships with all prehealth 
students, a number that can be as high as a few thousand in large universities. With that in mind, the advent of personal mentors into this equation opens doors to the world of complementary advising. General premed advising has demonstrated its ability to provide timely information on the current state of premed requirements, curriculum coordination, and a basic understanding of the application process for graduate school. It has proven less apt at directing students toward opportunities, academic and otherwise, that fit their near and long-term aspirations. The role of mentors in this coordinated effort is to further develop and direct some of the established student interests. The office of undergraduate advising should maintain a pool from a number of disciplines of viable mentors who could each advise a handful of students. Alternatively, students may identify their own mentor from within the university or perhaps from the community. In the end, some combination of this may be most easily achieved. A sample of this model is shown in Table 3.1.

As described above, this complementary system would not be modulebased, where students would change advisers when they advanced. Instead, the advising process evolves into a coordinated and dynamic structure capable of managing the multifaceted needs of prehealth students. In particular, this network of advisers could better identify the personality and interests of each student and develop plans (courses, extracurricular activities, and jobs or internships) that could be executed in a coordinated fashion with ease. In addition to students receiving a richer college experience and expanding their résumés, the increased exposure to advising or mentoring resources will be reflected in recommendation letters, calls to deans, and the content of the final application. Each member of this advisory system will play an integral role in ensuring that both near and long-term goals and requirements are met. Once the student's interests have been clearly defined, his or her mentor can effectively assist the student based on expressed interests and past activities.

Table 3.1. Roles in Advising Prehealth Students

General prehealth adviser

Concentration or major adviser

Mentor
Prehealth academic requirements, course scheduling, grad-school information, general reference for short-term undergraduate opportunities. Encourage early exploration of academic interests.

Information specific to undergraduate major (for example, chemistry, engineering, and so on)

Reference for trends in careers, industries. Contact for extracurricular and vocational activities. Ensures activities fit expressed interests and long-term goals. 
An alternative scenario might use a university or college's career planning and placement service for its upperclassmen. At this point, most prehealth students have finished a majority of course requirements and are focusing on standardized testing and applications, and some have even begun to seriously look into alternative career paths. Depending on the size and reach of this office, a portion of the burden may be able to shift onto the career planning office.

Clearly the long-term goal is to improve the quality of the prehealth advising process. Ultimately the advising that takes place is really a part of a much larger educational experience. Often, students have been forced to select a course of study or extracurricular activities early in their undergraduate experience to properly prepare for applying to graduate school. In some cases, this process actually begins years before college with advancedplacement examinations and math and science clubs and competitions. Needless to say, given the emphasis that students place on "getting in," it would seem worthwhile for advisers or mentors to make that a primary concern as well. That is, an appropriate advisory system may be able to better assist students in their quest for acceptance in the desired program because it has helped to create an individual, not simply a "premed."

\section{References}

Abdelman, W. H., Bryon, D. J., and Davidson, C. S. "A Useful Way to Orient Students to Medical Careers" (letter). Academy Medicine, 1995, 70, 83.

Alexander, S. F., Lyon, L. J., Nevins, M. A., Yere, L. R., Jr., and Thayer, H. S. "Ten Years of Orienting College Students to Careers in Medicine." Journal of the American Medical Association, 1992, 267(24), 3330-3331.

Alexander, S. F., Nevins, M. A., Lyon, L. J., Thayer, H. S., and Yere, L. R., Jr. "A Community Hospital's Program for Premedical Student Orientation." Journal of the Medical Society of New Jersey, 1983, 80(6), 421-422.

Becker, M. H., Katatsky, M. E., and Seidel, H. M. "A Follow-up Study of Unsuccessful Applicants to Medical Schools." Journal of Medical Education, 1973, 48, 991-1001.

Bruhn, J. G. "The Ills of Premedical Advising." Journal of Medical Education, 1977, 52, 676-678.

Bruhn, J. G., Fuentes, R. G., Jr., Trevino, F. M., and Williams, L. B., Jr. "Follow-up of Minority Premedical Students Attending Summer Enrichment Programs in a Medical Setting." Texas Medicine, 1976, 72(8), 87-96.

Caplan, R. M., Kreiter, C., and Albanese, M. "Preclinical Science Course 'Preludes' Taken by Premedical Students: Do They Provide a Competitive Advantage?" Academy Medicine, 1996, 71(8), 920-922.

Elam, C. T., Lenhoff, K., and Johnson, M. M. "Premedical Course Recommendations of Premedical Advisors, Medical Students and Medical School Faculty. Academy Medicine, 1997, 72(1), 72-73.

Gerbens, D. A., Stid, M. A., and Foulds, K. L. "A Collaborative Internship Program for Premedical Students" (letter). Academy Medicine, 1998, 73(8), 827-828.

Hesser, A., Cregler, L. L., and Lewis, L. "Predicting the Admission into Medical School of African American College Students Who Have Participated in Summer Academic Enrichment Programs." Academy Medicine, 1998, 73(2), 187-191. 
Imperato, P. J. "The Need for Premedical Curricular Reform" (letter, comment). Academy Medicine, 1997, 72(9), 734-735.

Stimmel, B., Smith, K., and Kase, N. "The Humanities and Medicine Program: The Need for the Traditional Premedical Requirements." Academy Medicine, 1995, 70(5), 438.

Trevino, F. M., Fuentes, R. G., and Bruhn, J. C. "The Premedical Advisory Process: Attitudes of Premedical Advisors. Texas Medicine, 1977, 73, 79-85.

TIMOTHY R. B. JOHNSON is chair and Bates Professor of Diseases of Women and Children in the Department of Obstetrics and Gynecology, Medical School, research scientist in the Center for Human Growth and Development, and professor in women's studies, College of Literature, Science and the Arts at the University of Michigan, Ann Arbor.

PHILIP D. SETTIMI is an honors economics graduate of The University of Michigan, Ann Arbor and a student at the University of Michigan Medical School.

JULIET L. ROGERS has an undergraduate degree in political science and a Masters degree in Public Health. She is a doctoral candidate in the Department of Health Management and Policy at the School of Public Health, University of Michigan, Ann Arbor and codirector at the Center of Excellence in Women's Health of the University of Michigan Health System. 